\title{
Development Assessment Centres: Practice Implications Arising from Exploring the Participant Voice
}

\section{Adam Turner, Health Education England (National Health Service, UK) Lynn Nichol, University of Worcester, $U K$}

\begin{abstract}
This qualitative phenomenological study explores the short-to-medium term personal impact of Development Assessment Centres on UK healthcare managers. The study identified overarching themes relating to personal performance impact, enabling and disabling factors in Centre design, trauma and safety implications, and behavioural adaptation. Practice implications arising focused upon three key areas. Firstly, Centre design should equally enable both introverts and extraverts and provide conscious consideration toward behavioural adaptation amongst participants. Secondly, there is a need for adequate follow-up support to enable participants to continue to learn from their experience, whilst also mitigating any potential risk toward long-term trauma caused by such deeply personal experiences. Finally, where assessment and reward form an output from any Centre, judgement should be limited until a thorough de-brief has been undertaken with the participant to explore causal behavioural responses, as opposed to basing decisions on observed behaviour alone.
\end{abstract}

\section{Introduction}

Empirical studies relating to the impact and validity of Development Assessment Centres (Centres) have been undertaken since the 1970s. However, these appear to have taken a predominantly positivist and quantitative perspective. Although valuable in providing generalisability surrounding the practice-based application of Centres, there appears a lack of understanding toward the personal and lived experiences of participants involved in such activities and any practice-based implications that this may reveal.

A methodological approach employing Interpretative Phenomenological Analysis (IPA) was used to enable the exploration of the personal impact of Development Assessment Centres upon a small sample of UK healthcare managers. IPA was specifically chosen to allow us to connect to the lived experiences of this sample group through its person-centric approach. The study seeks to compare and augment our existing knowledge and to explore the potential of gleaning an added layer of insight that this purely qualitative approach brings to the field. Most importantly, it considers how practitioners using such Centres could apply its findings within both development and assessment contexts.

\section{Development and Assessment Centres}

Assessment Centres have been utilized to test for specific role and promotion suitability since the late 1970s, with the majority of research having been undertaken since the 1990s. In recent years, Development Centres have become more commonplace in personal and career development (Goodge, 1994; Hart, 1979; Hinrichs, 1978; Thornton and Krause, 2009; Tillema, 1998; Vloeberghs and Bergham, 2003; Wilson, 1996). 
Although both approaches follow similar procedures and forms of assessment (e.g. psychometric tools, observed scenarios, in-tray exercises, multi-source feedback) there is general agreement that their underpinning purpose is different. Assessment Centres deductively assess for a specific suitability through a pass or fail approach, often testing for promotion suitability. Development Centres inductively generate self-insight to explore current performance, future potential and development gaps within a more nurturing environment. In all cases, there is a general consensus that the assessment and feedback aspects of Centres have a positive impact upon participants, which in turn increases both current performance and future potential (Arnold, 2002; Brits et al., 2013; Goodge, 1994 \& 1995; Halman and Fletcher, 2000; Iles, 1992; Jansen and Stoop, 2001; Ritchie, 1994; Vloeberghs, 2003; Wilson, 1996).

More recent thinking identifies the interconnected nature between assessment and development within these Centres. Goodge $(1994,1995)$ cited as authority in several papers (e.g. Arnold, 2002; Hetty-van-Emmerik, 2008; Vloeberghs and Bergham, 2003) proposed First, Second and Third Generational approaches to Centres, identifying a continuum of assessment-development. FirstGeneration is designed for assessment and selection only, Second-Generation for limited personal development and Third-Generation for pure development with subsequent developmental support.

In acknowledgement of the overlap between development and assessment, the term 'Development Assessment Centre' has become more widely used, acknowledging that this hybrid-fusion is superior, flexible and more cost effective in practice. For example, Centres are commonly used to simultaneously develop the organisational workforce whilst also identifying high potential candidates as part of a talent pool for targeted investment, development, or promotion (Brits et al, 2013; Hetty-van-Emmerik and Bakker, 2008; Jorgensen and Els, 2013).

Whilst examining empirical literature surrounding Centres, it was observed that there appears a lack of high-quality empirical research into the field that focuses upon the participants' personal perspectives of their experience, something that has also been cited by several authors (e.g. Arnold, 2002; Vloeberghs and Bergham, 2003). In terms of mixed methods perspectives, Arnold's work (2002) endorses the performance enhancement aspects of Centres and Francis-Smythe and Smith (1997) discuss the psychological impact of Centres upon participants. However, the opportunity to help shape and finesse practice by exploring the lived experiences of participants does not appear to be a predominant theme within wider empirical studies. Practitioners are currently missing important perspectives surrounding Centre design and application that could be gleaned from such a participant-centric enquiry.

\section{Research Question, Methodology and Sampling}

Seeking to explore the participants' lived experience of the Centre, this study sought to answer:

What are the personal perceptions of UK healthcare managers on the short-to-medium term impact arising from participation within Development Assessment Centres?

Interpretative Phenomenological Analysis (IPA) was chosen as a technique that is increasingly used within healthcare to explore the in-depth experiences of a homogenous sample group within a practice setting. IPA deliberately uses a small number of participants with similar characteristics to make accessible previously unexplored rich understandings of their individual experiences and actively enables and encourages the insider practitioner-researcher to immerse themselves into the practice setting. This approach allows us to gain a deeper interpretation/understanding 
surrounding the multiple individual perceptions of the sample group, acknowledging and emphasising the importance of the researcher as part of the interpretation, rather than as an objective bystander, whilst analysing the multiplicity of each individual's voice. Participant narratives are recorded, documented and thematically analysed to identify both common and unique insights (Costley et al., 2013; Smith et al., 2009).

Exploratory interviews took place with eight middle-management healthcare practitioners who had accessed the same style of Development Assessment Centre within the past year. This particular Centre was designed to bring together participants from multiple and diverse healthcare organisations and test their readiness to participate in a long-term and competitive leadership talent development programme through observed reactions to different leadershipfocused exercises and assessments against a healthcare leadership competency model. In all cases and regardless of pass or fail, participants were provided with feedback reports, coaching and signposted to wider personal development offers relevant to their assessment outcomes.

Participant narratives were separated into a pass and fail sub-group (Table 1) and thematically analysed to explore each participant's unique personal perspectives arising from their experience.

\begin{tabular}{lc}
\hline Participant Sample Description & 5 \\
\hline $\begin{array}{l}\text { Development Assessment Centre - Pass (P): Participants were successfully seen as } \\
\text { ready to progress onto a resultant leadership development programme. They gained } \\
\text { a findings report and participated in coaching feedback. }\end{array}$ & $\begin{array}{c}\text { Total Number } \\
\text { of Participants }\end{array}$ \\
$\begin{array}{l}\text { Development Assessment Centre - Fail (F): Participants were unsuccessful in } \\
\text { progressing onto a leadership programme. However, they did receive a findings } \\
\text { report, access to coaching feedback and signposting to alternative development. }\end{array}$ & 3 \\
\hline
\end{tabular}

Table 1: Sample Group Overview

\section{Analysis and Interpretation}

\begin{tabular}{|c|c|c|}
\hline \multicolumn{2}{|c|}{ Meta Theme } & Theme \\
\hline \multirow{8}{*}{$\begin{array}{l}\text { More present within } \\
\text { existing literature }\end{array}$} & \multirow{2}{*}{$\begin{array}{l}\text { A. Personal performance } \\
\text { impact }\end{array}$} & Self-awareness increases \\
\hline & & Changing, enabling and taking action \\
\hline & \multirow{4}{*}{$\begin{array}{l}\text { B. Enabling and disabling } \\
\text { factors }\end{array}$} & Enabling a feedback environment \\
\hline & & Need for on-going support \\
\hline & & Being ready for the experience \\
\hline & & Diversity of fellow participants \\
\hline & \multirow[t]{2}{*}{ C. Trauma and safety } & Anxiety \\
\hline & & Trauma and safety \\
\hline \multirow{2}{*}{$\begin{array}{l}\text { Less present within existing } \\
\text { literature }\end{array}$} & \multirow[t]{2}{*}{ D. Behaviour adaptation } & Acting and not being normal self \\
\hline & & Inhibiting for reflectors/introverts \\
\hline
\end{tabular}

Table 2: Summary of high level themes arising from analysis of interviews, presented in order of frequency observed within existing literature as reviewed during this research study 
This section reflexively synthesizes and interprets themes arising from participant narratives. Table 2 has been constructed to introduce the thematically summarized findings arising from the analysis of participant interviews during this empirical study. The ordering (A-D) relates to themes being more to less/not present within the existing empirical literature relating to Centres that was observed as part of the study.

The following section summarizes a detailed discussion surrounding the analysis and where appropriate, utilizes direct testimony from participant narratives to illustrate key points. We differentiate between those who passed (P) and those who failed (F) the Centres in these illustrations.

\section{A. Personal Performance Impact}

\section{Self-awareness increases}

Often expressed as 'emotional intelligence', Centres appeared to elicit strong feelings of becoming progressively self-aware, increased consideration towards personal impact upon others, and a higher level of confidence to 'be yourself'. Narratives identified how participants actively questioned these insights and how they could optimally apply them to increase their future performance. They linked this shift in self-awareness to the Centres' ability to offer constructive and real-time feedback through peer and facilitator observation. The combination of honest-feedback, space/time to think, and pure focus upon themselves rapidly allowed participants to take this self-insight to a deeper level.

Describing their stories appeared to induce a mixture of enlightenment and release, often evoking feelings of excitement and energy toward the emergent possibilities that the experience had given them. The experience appeared to help participants identify their personal voice, and at times gave them permission to be themselves, possibly due to the inductive and exploratory nature of the Centres. These findings support our existing knowledge that identifies the generic performance improvement aspects of Centres.

\section{Enabling, changing and taking action}

Self-insight appeared to transfer into enablement, generating a sense of achievement, which in turn positivity motivated and energized participants into taking action. This appears to expand upon Halman and Fletcher's (2000) findings whereby Centres elicit increased self-esteem.

Positive career impact was also a strong theme, building upon existing quantitative-empirical studies with similar findings (Hinrichs, 1978; Ritchie, 1994). Many participants gained new roles and the remainder were able to demonstrate increased responsibilities, including those who failed the Centre. Participants associated career progression to the insight, confidence and motivation generated by the Centres, demonstrated within the following narrative:

I then applied for a higher banded role. I'm now managing a national process, which I think before the assessment ... I wouldn't have felt confident enough to do (F).

In addition to career progression, narratives also demonstrated how participants were able to describe significant impact on their current job performance, which was often validated through feedback from others as demonstrated here: 
I've changed the way we go about discussing and creating ideas, it's more team led ... so we get a lot more ideas and innovation ... My manager has in fact noticed that it has improved me ... I can see myself I've changed (P).

These findings provide deeper knowledge towards supporting existing literature and also support the practical application of centres for both personal development within current role, and future career potential (Arnold, 2002; Brits, et al., 2013; Goodge, 1994 \& 1995; Iles, 1992; Jansen and Stoop, 2001; Jorgensen and Els, 2013; Vloeberghs and Bergham, 2003; Wilson, 1996).

Narrative surrounding an increased accuracy when identifying future development needs was also identified. This potentially appeared to relate to the impartiality of the evidence-base generated by the Centre, outside of any bias from a normal appraisal review. For this reason, participants from both the Pass and Fail groups identified how the experience acted as a development intervention in its own right:

Going through the Centre first, it pinpoints where your weaknesses are, so you can actually go on the course 'knowing' it's going to help you, rather than only 'thinking' it's going to help (P).

I actually think the Centre would be as good as attending a development programme to get people aware of what they need to develop in their future (F).

The rapidness of self-insight gained, increased motivation, and evidence-based accuracy of career next-steps is supportive of existing literature relating the use of Centres and creating Return-onInvestment (Thornton and Potemra, 2010). It therefore appears to endorse the practice-based use of Centres as cost-efficient, rapid development and performance enhancement interventions in their own right.

However, it was observed that the interchangeable assessment-development focus of the Centres appeared to subtly vary the participants' emotional responses toward their experience and resultant motivation to take further action. The assessment layer of the Centres appeared to evoke feelings of 'I had done my best', being 'good enough' and an emotional 'sigh of relief' that it was over. For some participants, this focus on purely passing the assessment aspects of the Centre appeared to take priority over any focus upon exploring/embracing the personal developmental aspects that the Centres were also designed to elicit. This provokes consideration toward the need for practitioners to ensure that follow-up support is provided to explore the developmental outputs from the Centres to maximize Return-on-Investment from the experience, as we will explore in more detail in sections $\mathrm{B}$ and $\mathrm{C}$.

\section{B. Enabling and Disabling Factors}

\section{Enabling a feedback environment}

Multiple-sources of clear impartial feedback, as identified as enabling by Goodge (1995) combined with good facilitation and the safe environment generated from a ground-rules / group-contracting phase enabled participants to become open to exploring diverse feedback during the experience:

[It] helped me understand what other people were thinking ... even if there's something there that you think isn't true or fair, the fact remains that it's somebody's interpretation of you ... take that on board ... that's obviously the impression that I gave to the person on the day, and need to think about how I come across to others $(\mathrm{P})$. 
The importance of good quality, encouraging, 'warm and friendly' facilitation was also highlighted as a key aspect toward creating this enabling environment.

\section{Need for on-going support}

Potentially relating to the rapidness of the experience, the need for on-going support to continue the journey of self-discovery resulting from the insights gained during the Centres appeared fundamental in the design of the Centres by all participants. This was encapsulated by one participant who articulated:

One day may not be quite enough ... it has such an impact on you, you need a way to digest it afterwards $(\mathrm{P})$.

This supports the stance of existing literature and best-practice guidelines suggesting that Centres should not be a standalone event (British Psychological Society, 2003 \& 2015; Goodge, 1995; Vloeberghs and Bergham, 2003). Although all Centres within this study offered feedback-coaching, participants who found ongoing methods to explore the learning arising from the experience in deeper levels of detail (e.g. coaching, manager-support, reflective exercises, wider development programmes) appeared to be the most successful in demonstrating subsequent personal impact. Congruent with many sectors, the majority of participants expressed healthcare as a high-pressured environment, identifying a risk that their self-insights gained could be 'placed on the shelf' without a form of on-going support, therefore reducing Return-on-Investment of the developmental activity.

Essentially the Centre signposted and opened doors, however responsibility was on the participant whether they chose to walk through such a door longer-term. Participants who did not embrace the support available to make-sense of the often-overwhelming amount of selfinsight gained, appeared least able to identify personal-impact. This appeared a lonely place for these individuals who needed further support, but for varied reasons appeared to have been apprehensive to seek it. As we will explore later, several participants appeared to close the door completely due to the intensity of their experience. It became clear that where participants had not found effective ways to make-sense of their experience, the research interview evoked a form of coaching conversation. Reflexively, this was an unexpected role that the interviewer realized they had taken on. One participant made direct reference to the interview being a sense of release, whilst another suggested 'keep asking questions, like you are now' as a means to help them gain post-Centre insight. This supports the significant need for practice to ensure participants are able to continue to make-sense of their experiences after the event.

Subsequently, the interviewer recommended coaching to several participants following the interview. It is interesting to reflect how participants appeared more engaged to participate in coaching after the interview, even though coaching formed the basis of the Centre and was offered to all as an ongoing development offer after the experience. This could be explained by the impartiality of the interviewer, allowing the participants to more easily open-up and see the benefit from exploring their experience further.

\section{Being ready for the experience}

Building upon the theme of needing post-Centre support, the concept of 'being ready' for the experience appeared critical as illustrated by one participant stating "it needs to be self-directed ... something you've got drive to do". However, from a practitioner perspective, assessing for 
prior readiness appeared paradoxical, as the intended outcome of the Centre is a readiness assessment itself.

Ultimately, being ready appeared to be seen as exhibiting an open-mind and a strong desire/ ambition for on-going self-discovery, as well as a drive for the reward of getting a place on the subsequent development programme. Testing out for these drivers when selecting participants to engage in similar Centres may be beneficial within the practice-based setting.

\section{Diversity of fellow participants}

The multi-professional environment of the Centres, bringing together participants from varied roles across healthcare appeared to empower participants to learn from each other's diverse professional perspectives. This diversity of exposure appeared unlikely to naturally occur within their day-to-day professional roles:

... engaging with people from other agencies ... get some common ground and build on it ... Before I attended if there was people I didn't know, I'd stay on the peripheral really ... it gave you those interpersonal skills from interaction with other agencies $(\mathrm{P})$.

Whilst exploring personal values elicited by the in-depth nature of the Centres, participants also appeared to establish an understanding that diversity transcends labels and began to see the positives in difference and learning from each other:

It was interesting to have a complete mix of people from different stages in their careers and different roles ... recognising everyone's priorities and strengths, to enable us, in recognition of our differences and using it as a positive (P).

This could suggest that Centres may actively support the practice of organisational diversity (i.e. we are all different) and inclusion (i.e. celebrating and valuing individuals' difference to help people reach their potential) agendas, which are areas of business responsibility that appear increasingly linked to HR practice functions.

Building upon this observation, participants appeared to positively learn from each other by observing the diversity of their peers' behaviours, identifying the enabling and disabling features congruent with their own personal values and beliefs. For some, this learning provoked a choice towards an increased or decreased demonstration of this behaviour in their own future-self. Whilst in these instances, the behavioural focus generated positive self-insight, section D will explore the more complex issues observed surrounding participant behaviour.

\section{Anxiety, Trauma and Safety}

Contrasting with the generic enabling themes identified above, a polarising theme arose whereby the assessment-focused environment had the potential to cause levels of trauma. Although all participants identified levels of 'anxiety', 'nerves' and 'exhaustion', more traumatic side-effects were identified by participants, provoking survival and fight-or-flight responses. This was seen in the majority of participant narratives, both those who passed and failed the Centres. However, it appeared particularly heightened for those who failed and/or had unmet self-reflection needs. The following participant, who did pass, identified how certain exercises elicited a direct form of personal trauma: 
[It was] ... one of the most intense days of my life. It sounds like a really dramatic thing to say (laughs) but it really was ... When we did that exercise, I absolutely hated that. Every fibre of me wanted to run away ... I made up my mind if I was asked to do anything like that again, I'd say no (P).

In this case, the participant went on to describe how they were able to translate this experience into a positive outcome. In other cases, the experience appeared to shut-down participants who subsequently sought no further support to make sense of their experience, resulting in levels of what appeared to be longer-term trauma.

Although feedback reports, coaching and wider development offers were provided to all participants, this further heightens the need for targeted post-Centre engagement and support, highlighted by the following participant narrative:

If their confidence is lacking and they had a bad experience, you don't want it to take you down even lower ... obviously you need to have the support mechanisms in place afterwards if needed (P).

Exploring the narratives of individuals that felt disabled by the Centre, it potentially appeared that the Centres were also evoking levels of unresolved past-life trauma, further inhibiting their performance. One participant who subsequently failed the Centre described feeling shut-down and unable to positively participate from perceived criticism. Another described how an initially positive experience turned intensely traumatic as a result of an exercise that appeared to bring back memories of historic trauma. This resulted in a loss of self-control, impeded performance, and was directly attributed to personal failure in the Centre environment:

I'm quite an intrinsically motivated person. As soon as something knocks me off my kilter I find it hard to get back ... the exercise made me feel out of control ... I actually work in a very deprived area where trauma is part of people's everyday lives ... looking after families or mothers or whatever, who have had these traumatic experiences ... I don't know how to explain it really. I don't think they realized that what [the exercise] means to you $(F)$.

Although levels of mild trauma could be seen in all participant narratives, this deeper level of performance inhibiting trauma only appeared in participants who failed the Centre. For these individuals and potentially resulting from the sense of being unable to deal with this trauma in the moment, feelings appeared to manifest themselves in denial and an inability to express any positive impact from the Centre experience. However, exploration during both interviews did elicit evidence of positive performance improvements, but to a lesser degree than those who had passed. These participants appeared to shutout the experience as the only means to deal with it.

These findings potentially build-upon insights within the studies by Hetty-van-Emmerik and Bakker (2008) and Francis-Smythe, J., and Smith (1997) identifying how negative or poor quality feedback from Centres, coupled with work pressures has a direct impact upon the participants' psychological state and is likely to lead to negative future performance implications.

Building upon findings within section A, one participant who failed the Centre named the research interview experience as 'cathartic'. This sense of release possibly demonstrated how, although a year had passed, the exploratory questioning during the interview had allowed adequate reflexively into exploring deeper self-insights, thus becoming more open to learning from the experience. As mentioned previously, when coaching was offered after the interview, participants were open to this, potentially as a result of the impartial and exploratory questioning during the interview. Again, on-going post-Centre support appears ethically critical within the practice of running these Centres. 


\section{Behavioural Adaptation}

\section{Acting, not being normal self}

Several participants innocently described experimenting with new behaviours during Centres, as demonstrated here:

I thought of it as a different side of me really ... I was enjoying a new confidence to speak out and direct things ... I think the Centre brought me out of myself really (P).

Two possible scenarios appeared to arise here. Firstly, Centres may have given participants permission to identify and test out new behaviours that better resonated with their unique values, the confidence to keep them, and thus demonstrate a truer version of themselves. Alternatively, Centres may have directed participants towards acting out certain behaviours during the Centre, eliciting a learnt behaviour rather than being a true reflection of who they really are. Reflexively, both scenarios appeared to be taking place.

Building upon these observations further, a concerning theme of being unable to be your normalself and more disturbingly learning how to behave to pass arose, potentially as a result of being driven towards the reward for passing the Centre. This was demonstrated within the following participant narrative, who did indeed pass:

It took me a little part of the morning to actually twig, how to behave ... the first bit was actually trying to understand it $(\mathrm{P})$.

If accurate on a wider scale, these findings may have significant validity implications for assessmentfocused Centres. It would appear to contradict the rationale for the Centres pass-or-fail assessment environment, which has the intention of eliciting behaviours to predict future performance and therefore potential. These findings appear amplified when coupled with the following theme that identified how the Centres appeared inhibiting toward more reflective and introverted participants.

\section{Inhibiting for reflectors/introverts}

For participants with clearer self-identified introverted and reflective personality preferences, the intense assessment focused environment with little time for self-reflection appeared to make them feel forced to adapt and behave extraverted to be seen, observed and assessed. For these participants, the Centre proved overwhelming and subsequently appeared to disadvantage this audience. This generated a sense of frustration and being torn-apart. More notably, all three participants that failed the Centre identified themselves as introverts/reflectors:

I already knew I'm a reflector ... I found it quite intimidating because I like to reflect on things before I put my opinion, and I felt that wasn't taken into account more, so I felt on the back-foot with some groups I was in because there were such big personalities ... I was trying to adapt my learning style to fit in with everyone else's, rather than mine being incorporated into the day $(\mathrm{F})$.

Although the above narrative identifies pre-awareness of their reflective preferences and feelings of being disadvantaged due to this, they later went on to describe significant post-Centre achievement. They sought out alternative self-directed learning opportunities and development programmes, obtained a senior national promotion, and directly linked this back to the personal insights that the Centre gave them. 
This begins to question how, despite failing the Centre, did the individual achieve this as a result of the Centre experience? Or alternatively, did the Centre environment disadvantage them because of their reflective preferences, and in fact should have identified that they were ready to progress? However, this individual worked within an educational environment and therefore may have had increased access to developmental support to enable wider reflection and positive actions after the experience.

It should be noted here that whilst the two other participants who failed the Centre were able to identify increased role responsibility, neither directly related this to the outcomes from the Centre. It should also be noted that this observation of being forced to become seen was also identified within participants who passed the Centre: “... they were just saying things to be heard, because it was a bit of an artificial situation" (P).

Some participants who appeared to exhibit introverted/reflective preferences and the subsequent inability to consciously adapt their behaviours went on to describe seeing peers compromise their values to adapt their behaviours for the reward of passing. This feeling appeared strongest in those who failed the Centre:

I'm intrinsically motivated, when I looked at other people and even though they were unhappy about the task ... they were going to participate even though they were unhappy about it, the sort of extrinsic thing ... the reward for getting it ... they will actually jump through hoops ... to achieve their goal ... compromising your own integrity slightly to do that (F).

The values-based dilemma described by this particular participant was so overwhelming that a sense of disapproval toward their peers appeared to overshadow any possible positive impact for themselves. This may potentially demonstrate a lack of open-mindedness/readiness for the Centre, identified as a critical enabler above in section A. Alternatively, the experience itself may have caused animosity toward their own lack of skill to adapt their behaviours. Perhaps by perceiving others as appearing to compromise their values to pass and seeking to blame them was in fact a mechanism to ease this personal discomfort. Either way, this discredited the Centre in the eyes of that participant causing them to give up on the experience. Reflexively considering the participant interviews, all of these factors may have been at work.

This values-based choice; to compromise beliefs and natural behaviours to 'be seen' and risk reduced performance by acting outside of behavioural preferences, or to remain their true-self and risk failing the Centre, appears in direct conflict with the previous enabling theme of gaining your voice. Here, Centres appeared to take the voice away from these participants, ultimately resulting in an intense sense of letting themselves down due to this inability to adapt behaviour as identified by the following participant:

[I felt] a little bit demoralized ... like I'd let myself down, when I knew the answers really, I just hadn't got the assertiveness to say them $(\mathrm{F})$.

Synthesising the above themes, both ethical and validity considerations arise, which appear lacking within Centre best-practice guidelines (British Psychological Society, 2003; International Task-Force, 2009). It appears that the assessment and reward focused aspects of these Centres may have inadvertently assessed some individual's abilities to identify and adapt to the required behaviours to pass, rather than assessing their natural behaviour. This caused a proportion of participants to act out of character and not allow observation and assessment of their natural behaviour. Coupled with potentially disadvantaging those with introverted and reflective 
personality preferences who are less able to rapidly adapt to the Centre, this ultimately calls into question; are we really assessing true performance and potential, or are we simply assessing the ability of participants to identify the required behaviours to pass and to successfully act into these? It also appears that this drive to pass the assessment aspects and resultant reward could be taking precedence over embracing the developmental aspects and self-insight that could be gained through approaching the Centre by behaving as their normal self.

This potentially unforeseen impact generated by the assessment-focused aspects of the Centres warrants further investigation on a wider scale, particularly in relation to using Centres for assessment within role progression, recruitment purposes, or where any form of reward is offered. Furthermore, it draws the validity of such Centres into question with due consideration towards fair Centre design when assessing reflective and introverted participants, especially when based on pure assessor observations alone. It also provokes consideration as to whether we, as practitioners, should combine both assessment and developmental aspects of Centres, if any assessment focus appears to potentially undermine the ability of some participants to embrace developmental aspects.

\section{Discussion}

\section{Summarising key findings from participant narratives}

The participant focused perspective of this study appeared to be supportive of and augment our existing knowledge observed within existing empirical research, identifying that Centres can enable participants to generate self-insight and enhance personal performance within their current role, whilst also providing support toward identifying and exploring future career potential.

However, the study also generated wider insights surrounding behavioural adaptation, potentially disadvantaging participants with introverted and reflective personality preferences, and most concerning the potential for the experience to induce trauma. This alternative picture directly contrasts with the majority of existing studies that demonstrate Centres ability to positively enhance performance and potential.

A values-based choice appeared to present itself for those participants who felt unable to behave as their normal selves. They could try and adapt their behaviour to be seen/survive the experience, risking performance degradation due to being forced outside of their natural behavioural preferences and comfort zone, or alternatively choose to not be seen at all and risk failing the Centre completely.

Negating the Centres purpose to assess future performance and readiness for career progression, an interrelated theme arose whereby individuals appeared to learn how to behave to pass, rather than behaving as themselves, driven by the rewards that passing the assessment aspects of the Centre would offer. These observations potentially mean that Centres could be assessing the ability of the more astute participant to identify and adapt their behaviours to pass, therefore raising validity challenges in Centre application.

For individuals who could not adapt their behaviour to be seen, coupled with the potential for certain exercises to provoke high levels of trauma, the Centres became overwhelming, and effectively these participants shut-down from the experience both during and after the 
Centre. This resulted in them being less likely to embrace post-Centre support, and effectively induced a layer of unresolved trauma. Although a mild level of performance improvement was described, disturbingly this increased level of trauma appeared to be potentially inhibiting future performance for these participants, rather than enabling them to reach their potential.

Considerations toward behavioural adaptation, balancing introverted/extraverted preferences and mitigating trauma do not appear substantially accounted for within existing literature, or best-practice ethical guidelines for facilitating Centres (British Psychological Society, 2003; International Task-Force, 2009). This means that considerations toward these areas may be overlooked within the design, validity and ethical practice within existing Centres.

\section{Practice implications}

Findings are congruent with wider studies that see the benefits of using the development and assessment nature of the Centres to rapidly identify and signpost individuals toward their future potential. The study also endorses the use of Centres to increase Return-on-Investment in staff development, providing an evidence-based view towards the most appropriate development interventions for participants. This should be equally considered in the context that many participants saw the rapid development provided through the self-insight gained during the Centre as all they needed. Even those who had failed could identify performance improvements and even promotions that they related to the insights gleaned from the experience. An interesting application of Centres being used to support organisational diversity and inclusion agendas also arose.

Using Centres as part of an internal talent strategy may therefore potentially reduce the resource burden associated with placing large proportions of staff on longer-term development programmes. In addition, it may endorse the use of Centres in helping individuals to identify their potential and enhance the accuracy of signposting them to their next developmental steps by removing the bias from career conversations which are most likely to take place within an appraisal setting. This increased accuracy may therefore increase Return-on-Investment for any subsequent organisational investment in these individuals.

There also appears to be a darker side to the use of Centres in practice when combining assessment and development aspects. Whilst this study cannot state generalizability, it may provoke careful choices for practitioners when hybridising development and assessment aspects within Centres. Whilst existing literature identifies that practice often combines development and assessment due to resource and funding limitations (Goodge, 1995; Vloeberghs and Bergham, 2003) practitioners may wish to consider how the assessment focus of any such Centres could have profound and negative impacts upon some participants. This appears particularly true for those with introverted and reflective preferences and unresolved past-life trauma, the latter being almost impossible for practitioners to know about.

More worryingly the potential for some participants to actively choose to change their behaviour in order to be seen in a way that enables them to pass appears to invalidate the use of the Centres altogether. These aspects may therefore result in imprecise assessment, identifying less suitable candidates for any subsequent reward, as well as rendering any personal development aspects arising from the Centre as inaccurate or of less value for participants. All of this in turn could reduce the Return-on-Investment for the participants and for the organisation investing in the Centres. 
Ensuring quality post-Centre feedback and placing strict emphasis on a wider form of follow-up support such as coaching appear ethically essential within such assessment-focused environments where participants have reduced time to think and reflect. This will ensure that any potential level of anxiety or trauma elicited by Centres can be mitigated and addressed, and also enables maximum Return-on-Investment toward the developmental aspects that Centres provide participants.

Findings would also suggest that where any form of assessment takes place, exercises should be cognisant towards allowing introverts/reflectors to feel able to participate equally and be seen. As part of Centre design and to increase validity of outcomes, practitioners may consider integrating ways to measure and test that participants are indeed behaving as their natural self and congruent with their personal values, or if they are adapting their behaviours driven by a need to pass. Practitioners may wish to consider how exercises can continually mix combinations of competencies to ensure participants are less likely to realize how to behave to pass and act into this. Allowing for exercises that equally advantage introverted/reflective participants, for example, individualized scenarios and one-to-one interpersonal tasks, may also enable these types to feel more able to participate, feel seen and therefore see the experience positively. The use of psychometrics to identify personality preferences in advance of the Centre may also aid facilitators in contextualising their observations of participants during exercises. However, this would increase resource implications whilst running Centres.

It is also recommended that any judgement on pass or fail should be withheld until a thorough de-brief has been undertaken with the participant, rather than making any judgement purely on observed behaviour alone. This de-brief should be designed to explore and understand why the participant approached the Centre the way they did, what was going on for them internally that couldn't be observed, how this related to their personal values, past experiences and also behavioural preferences. Essentially this form of post-Centre feedback coaching could form the final part of the assessment. This in turn would allow space for the participant to further explore their experience in a developmental context, be supportive toward participants with reflective preferences, reduce any potential trauma elicited, promote the positive developmental aspects of the experience and focus the participant on moving-forwards toward their potential.

Findings from this study may also question the use of pure Assessment Centres in practice. For example, within job applications, promotions and where any form of reward is offered. Practitioners may wish to consider the transferability of the findings from this study in these contexts, and ensure that any assessment environment adequately allows participants of both introverted, extraverted and wider personality preferences the ability to demonstrate their potential fairly and equally, with additional time allocated for a formal debrief/feedback coaching as part of the assessment. Without consideration towards this, practitioners may be limiting their choice of candidate to those who can 'shout the loudest' or those who can simply adapt their behaviours to what is being tested, rather than truly identifying the best candidate for the opportunity.

\section{Research Implications}

Understanding that phenomenological studies do not seek to generalize and acknowledging that this is a small-scale study, it is strongly recommended that the tensions identified here are further studied across a wider cross-section of Centres. In particular, contrasts between both assessment and development-focused environments should be considered. 
The additional facets of understanding in the context of existing research, together with the new perspectives demonstrated within this study also confirm the added value that a qualitative research paradigm can bring towards exploring the field of Development and Assessment Centres in the future.

\section{Conclusion}

This study was designed to develop a rich understanding of participants' individual lived experiences of Development Assessment Centres to provoke questioning of existing practice and discover potentially new avenues of research. Whilst supporting the stance of many existing empirical studies on the positive aspects of Centre application to enhance performance and potential, the exploration of participants' 'individual voices' in this study, has generated a set of findings with important implications for existing practice and new avenues for future enquiry.

Most notable of these findings include the potential for Centres to induce trauma and disadvantage some participants, particularly those with introverted and reflective preferences. Coupled with the possibilities that some participants may be actively adapting their behaviour for the reward of passing, rather than allowing observation of themselves in their natural state also brings validity challenges for future Centre application in practice. Whilst the study cannot claim these findings as generalizable, these themes can now be taken forward in subsequent studies to help develop more nuanced and effective approaches to enhance practice application of Development Assessment Centres.

\section{References}

Arnold, J. (2002). Tensions between assessment, grading and development in development centres: A case study. International Journal of Human Resource Management, 13 (6) 975-991.

British Psychological Society, (2003). Design, Implementation and Evaluation of Assessment and Development Centres: Best Practice Guidelines. The British Psychological Society.

British Psychological Society, (2015). The design and delivery of assessment centres: A standard produced by the British Psychological Society's division of Occupational Psychology. The British Psychological Society.

Brits, N., Meiring, D., and Becker, J. (2013). Investigating the construct validity of a development assessment centre. Journal of Industrial Psychology, 39 (1).

Costley, C., Elliott, G., and Gibbs, P. (2013). Doing work based research: Approaches to enquiry for insider-researchers. London UK, SAGE Publications Ltd.

Francis-Smythe, J., and Smith, M. (1997). The psychological impact of assessment in a development center. Human Relations, 50 (2), 149-167.

Goodge, P. (1994). Development centres: guidelines generation and effectiveness. Journal of Management Development, 13 (4), 16-22.

Goodge, P. (1995). Design options and outcomes: progress in development center research. Journal of Management Development, 14 (8), 55-59.

Halman, F., and Fletcher, C. (2000). The impact of development centre participation and the role of individual differences in changing self-assessments. Journal of occupational and organizational psychology, 73, 423-442.

Hart, G., and Thompson, P. (1979). Assessment centers: for selection of development? Organizational Dynamics, Spring Issue, 63-77.

Hetty-van-Emmerik, I., and Bakker, A. (2008). What happens after the developmental assessment centre? Employees' reactions to unfavourable performance feedback. Journal of Management Development, 27 (5), 513-527. 
Hinrichs, J. (1978). An eight-year follow-up of a management assessment center. Journal of Applied Psychology, 63 (5), 596-601.

Iles, P. (1992). Centred of Excellence? Assessment and development centres, managerial competence and Human Resource Strategies. British Journal of Management, 3, 79-90.

International Task Force on Assessment Center Guidelines (2009). Guidelines and Ethical Considerations for Assessment Centre Operations. International Journal of Selection and Assessment, 17 (3).

Jansen, P., and Stoop, B. (2001). The dynamics of assessment center validity: results of a 7-year study. The Journal of applied psychology, 86(4), pp. 741-753.

Jorgensen, L., and Els, B. (2013). Efficacy Evaluation of a Leadership Development Assessment Centre For Managers. Journal of Psychology in Africa, 23 (1), 113-117.

Ritchie, R. (1994). Using the assessment center method to predict senior management potential. Consulting Psychology Journal, 46 (3), 16-13.

Smith, J. A., Flowers, P., and Larkin, M. (2009). Interpretative Phenomenological Analysis - Theory, Method and Research. London UK, SAGE Publications.

Thornton, G., and Krause, D. (2009). Selection versus development assessment centers: an international survey of design, execution, and evaluation. The International Journal of Human Resource Management, 20 (2), 478-498.

Thornton, G., and Potemra, J. (2010). Utility of assessment centre for promotion of police sergeants. Public Personnel Management, 39 (1), 59-69.

Tillema, H. (1998). Assessment of potential, from assessment centres to development centres. Assessment of Potential, 6 (3), 185-191.

Vloeberghs, D., and Bergham, L. (2003). Towards an effectiveness model of development centres. Journal of Managerial Psychology, 18 (6), 511-540.

Wilson, D. (1996). The future of development centres. Career Development International, 1 (6).

\section{The Authors}

Adam Turner is Leadership and Organisational Development Lead for Health Education England. He has worked in the field of organisational development for the entirety of his career and is passionate about developing people, organizations and systems. He has a keen interest in the field of talent management, having previously led nationally on the NHS Leadership Academy talent management programme of work, developing healthcare talent toolkits and approaches that have been rolled out nationally across the UK NHS. Adam holds a Masters degree and is currently studying towards his $\mathrm{PhD}$ at the University of Worcester.

Dr Lynn Nichol is a Principal Lecturer in HR and Head of the Leadership, Management and HR Subject Group at the University of Worcester. She is committed to developing organizational practice, through research and leads the Professional Doctorate and DBA programmes at the University. She works with senior practitioners from a wide range of organizations seeking to influence and change practice through their insider research. Her current work focuses on how managers make use of academic scholarship, work based learning and using mentoring and coaching in doctoral supervision. 